\title{
BIBECHANA
}

A Multidisciplinary Journal of Science, Technology and Mathematics

ISSN 2091-0762 (online)

Journal homepage: http://nepjol.info/index.php/BIBECHANA

\section{Effect of solvent composition on the critical micelle concentration of sodium deoxycholate in ethanol-water mixed solvent media}

\author{
Ajaya Bhattarai*, Sujit Kumar Shah, Ashok Kumar Yadav, Janak Adhikari \\ Department of Chemistry, Mahendra Morang Adarsh Multiple Campus, Tribhuvan University, \\ Biratnagar, Nepal \\ * Corresponding author: E mail: bkajaya@yahoo.com \\ Article history: Received 18 October, 2012; Accepted 3 November, 2012
}

\begin{abstract}
The precise measurement of the specific conductivity of sodium deoxycholate in pure water and ethanolwater mixed solvent media containing 0.10 and 0.20 volume fraction of ethanol at $303.15 \mathrm{~K}$ are reported. The concentration were varied from $\sim 0.01 \mathrm{~mol} \mathrm{~L}^{-1}$ to $\sim 0.0002 \mathrm{~mol} \mathrm{~L}^{-1}$. The conductivity of sodium deoxycholate decreases with the increase in the volume fraction of ethanol. The critical micelle concentration of sodium deoxycholate increases with the increase in the volume fraction of ethanol.
\end{abstract}

Keywords: sodium deoxycholate; conductivity; critical micelle concentration; ethanol-water mixed solvent media

\section{Introduction}

Surfactants are organic substances, which significantly decrease the surface tension of water at relatively low concentrations, are at least partially water soluble. Surfactants are amphiphilic materials containing both a polar long-chain hydrocarbon "tail" and polar, usually ionic, "head" groups. In polar solvents, for example water, this dual character of the amphiphile leads to self-association or micellization, the surfactant molecules arrange themselves into organized molecular assemblies known as micelles. The hydro-phobic part of the aggregate forms the core of the micelle, while the polar head groups are located at the micelle-water interface in contact with and hydrated by a number of water molecules. Depending on the chemical structure of the surfactant, its micelle can be cationic, anionic, ampholitic (zwitterionic), or nonionic. When dissolving them, after they reach a certain value of concentration, molecules or ions of surfactants begin to associate and to organize themselves into micelles. In colloidal and surface chemistry, the critical micelle concentration $(\mathrm{cmc})$ is defined as the concentration of surfactants above which micelles form and almost all additional surfactants added to the system go to micelles [1]. Critical Micelle Concentration values are important indicators when considering which surfactant will provide optimal performance benefits. The determination of cmc is generally based on the localization of the position of a breaking point in the concentration dependencies 
of selected physical or chemical properties of surfactant solutions. Because of the surface activity of these substances, measurements of the surface tension of surfactant solutions represent the principal method of cmc determination. However, it is rather tedious and time-consuming procedure. In the case of ionic surfactants, the utilization of electrochemical measurement is much more convenient, especially the measurements of the electrical conductivity of their solutions with varying concentration. The conductometric method is based on the finding of a breaking point on the curves, which describe the concentration dependence of conductivity [2]. It is well-known, that the conductivity of any solution is directly proportional to the concentration of its ions. The point, where the micelle formation starts, is indicated on the concentration dependence of specific conductivity $(\mathrm{K})$ as a breaking point. It is easy to find the breaking point, because it marks a significant change of the linear slope of the dependence $\mathrm{K}=\mathrm{f}(\mathrm{C})$. The requested value of $\mathrm{cmc}$ is the intercept of two linear functions with mutually different slopes.

Sodium deoxycholate (fig.1), the sodium salt of deoxycholic acid, is a water-soluble, bile-acid, ionic detergent commonly used in protein methods. It is most frequently used as a component of cell lysis buffers (e.g., RIPA buffer), but also has been used for liposome preparation, isolation of membrane proteins and lipids, preventing nonspecific binding in affinity chromatography and a cell culture media supplement.

Deoxycholic acid has been used since its discovery in various fields of human medicine. In the human body deoxycholic acid is used in the emulsification of fats for the absorption in the intestine. It has, in some countries (including Switzerland) been licensed as an emulsifier in food industry [3].

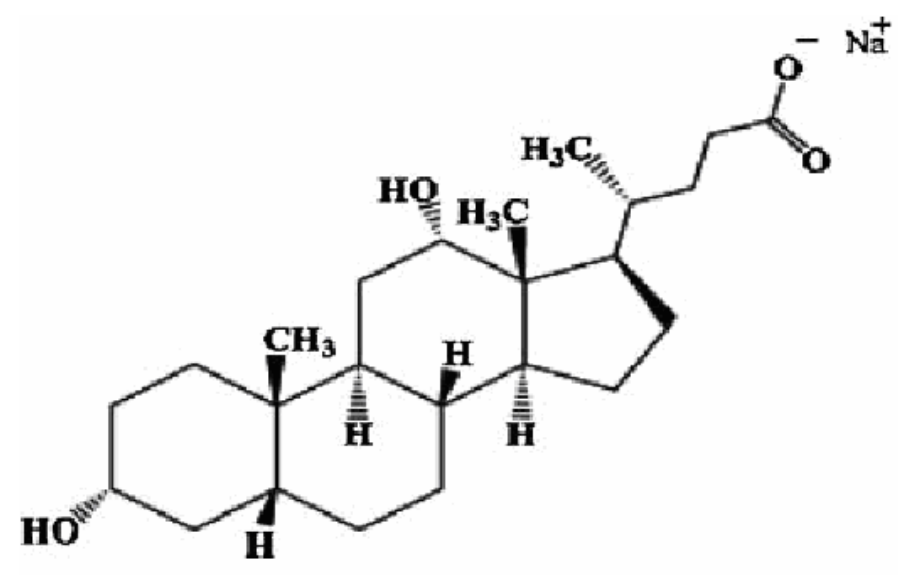

Fig.1: Structure of sodium deoxycholate.

Our aim of this paper is to determine the $\mathrm{cmc}$ of SDC in pure water and ethanol-water mixed solvent media containing 0.10 and 0.20 volume fraction of ethanol at $303.15 \mathrm{~K}$ by conductometric method.

\section{Experimental Methods}

Ethanol (E. Merck, India) was used for the experimental works. The purified solvent had a density of $0.78097 \mathrm{~g} . \mathrm{cm}^{-3}$ and a co-efficient of viscosity of $0.9490 \mathrm{mPa} . \mathrm{s}$ at $303.15 \mathrm{~K}$; these values are in good agreement with the literature values [4]. Distilled water with a specific conductance less than $10^{-6} \mathrm{~S}_{\mathrm{cm}}{ }^{-1}$ at $303.15 \mathrm{~K}$ was used for the preparation of the mixed solvents. The physical properties of Ethanol-Water mixed solvents used in this study at $303.15 \mathrm{~K}$ were taken from the published works [5-8]. 
Conductance measurements were carried out on a Pye-Unicam PW 9509 conductivity meter at a frequency of $2000 \mathrm{~Hz}$ using a dip-type cell with a cell constant of $1.15 \mathrm{~cm}^{-1}$ and having an uncertainty of $0.01 \%$. The cell was calibrated by the method of Lind and co-workers in 1959[9], using aqueous potassium chloride solution. The measurements were made at $303.15 \mathrm{~K}$ in a jacket containing conductivity cell of cell constant $1.002 \mathrm{~cm}^{-1}$. Water was circulated in the jacket from thermostat and the temperature was maintained within $\pm 0.1^{\circ} \mathrm{C}$. The details of the experimental procedure have been described earlier [10-11]. Several independent solutions were prepared and runs were performed to ensure the reproducibility of the results. Due correction was made for the specific conductance of the solvent by subtracting the specific conductance of the relevant solvent medium from those of the electrolyte solutions.

In order to avoid moisture pickup, all solutions were prepared in a dehumidified room with utmost care. In all cases, the experiments were performed in three replicates.

\section{Result and Discussions}

Conductivity measurements were performed in pure water and ethanol -water mixed solvent media at $303.15 \mathrm{~K}$ in order to evaluate the $\mathrm{cmc}$ of Sodium deoxycholate. The cmc of Sodium deoxycholate found at different volume fraction of ethanol -water mixed solvent media are listed in Table 1.The cmc of Sodium deoxycholate in pure water was found to be $2.04 \mathrm{mM}$ by fluorescence measurements at 298.15 $\mathrm{K}$ [12] which is almost matching with static light scattering measurements[13]. As the temperature increases of the solution of Sodium deoxycholate in water, the cmc also increases[13-14] and this is the best evidence for our conductivity measurements of Sodium deoxycholate in pure water.

The experimental specific conductivities of Sodium deoxycholate as a function of the its molar concentration at $303.15 \mathrm{~K}$ of pure water and two different ethanol-water mixtures (containing 0.10 and 0.20 volume fractions of Ethanol) are depicted in fig. 2 to 4 . It was observed that $\mathrm{cmc}$ of Sodium deoxycholate increases with the increase in the volume fraction of ethanol. This trend is due to decrease in the relative permittivity of the medium. The relative permittivity of the medium decreased at a given temperature with increasing in the Ethanol content and similar trends have been observed in the previous works [6-7]. The presence of Ethanol reduces the dielectric constant of the solvent phase and makes easier for the formation of ion-pairs in the solution phase.

It is also well known that addition of solvent which acts as water structure breakers decrease the hydrophobic effect result in to the increase in the cmc value of ionic surfactants [15]. Breaking of water molecules by ethanol molecules would facilitate interation between the hydrophobic tail of the surfactant molecule and the hydrophobic part of the ethanol and consequently the local concentration of ethanol molecules around the surfactants monomers becomes larger than the bulk solvation of the surfactant molecules by the hydrophobic part of the organic solvent would therefore lead to the delay aggregation of the surfactant monomers to form micelle and hence the increase in the cmc of the surfactant [16].

Table 1: The Critical Micellar Concentratiom (cmc) obtained from Conductometry of SDC in Pure water and Ethanol-Water Mixed Solvent Media Containing 0.10, 0.20 volume fraction of Ethanol at 303.15 K

\begin{tabular}{|l|l|l|l|l|}
\hline $\mathrm{T}(\mathrm{K})$ & $\begin{array}{c}\text { water } \\
\mathrm{cmc} \\
(\mathrm{mM})\end{array}$ & $\begin{array}{l}0.10 \\
\text { ethanol } \\
\mathrm{cmc} \\
(\mathrm{mM})\end{array}$ & volume fraction of & $\begin{array}{l}0.20 \text { volume fraction of ethanol } \\
\mathrm{cmc} \\
(\mathrm{mM})\end{array}$ \\
\hline 303.15 & 2.91 & 3.74 & 4.15 \\
\hline
\end{tabular}


A. Bhattarai et al. / BIBECHANA 9 (2013) 63-68 : BMHSS, p. 66 (Online Publication: Nov., 2012)

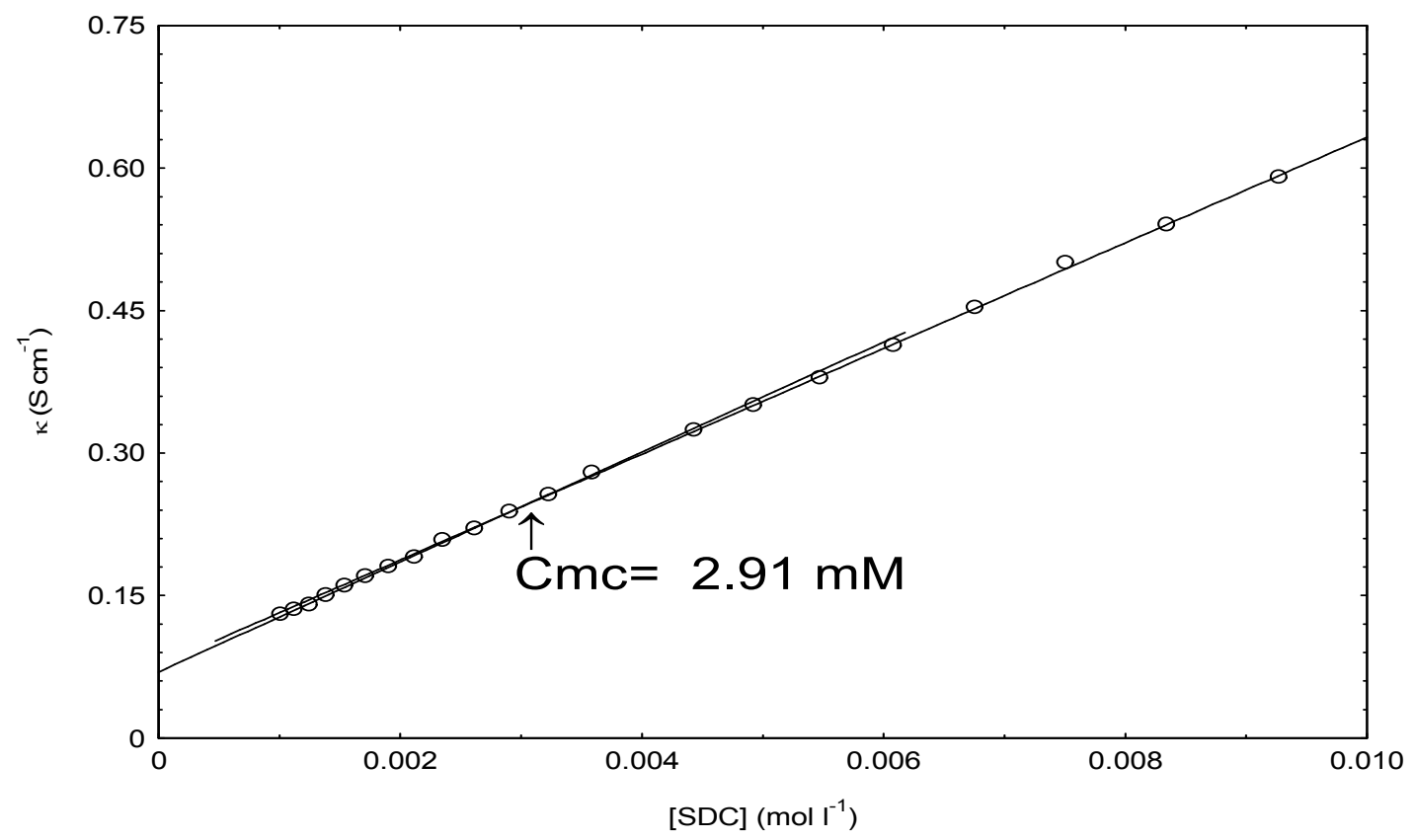

Fig. 2: Specific conductivities of SDC as a function of the surfactant concentration in $303.15 \mathrm{~K}$ : opened circles represent pure water.

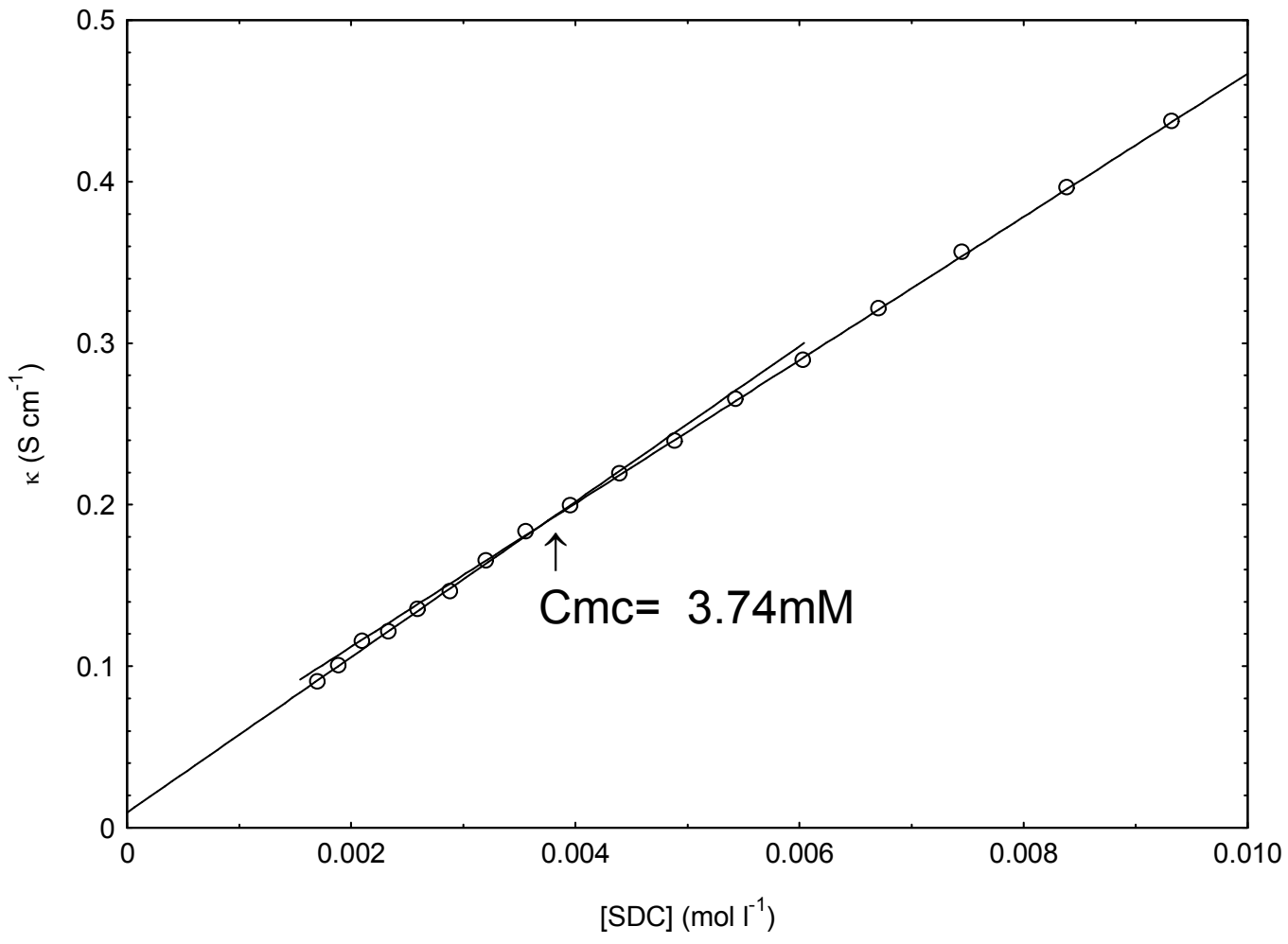

Fig. 3: Specific conductivities of SDC as a function of the surfactant concentration in $303.15 \mathrm{~K}$ : opened circles represent 0.10 volume fractions of ethanol in the solvent mixture. 
A. Bhattarai et al. / BIBECHANA 9 (2013) 63-68: BMHSS, p. 67 (Online Publication: Nov., 2012)

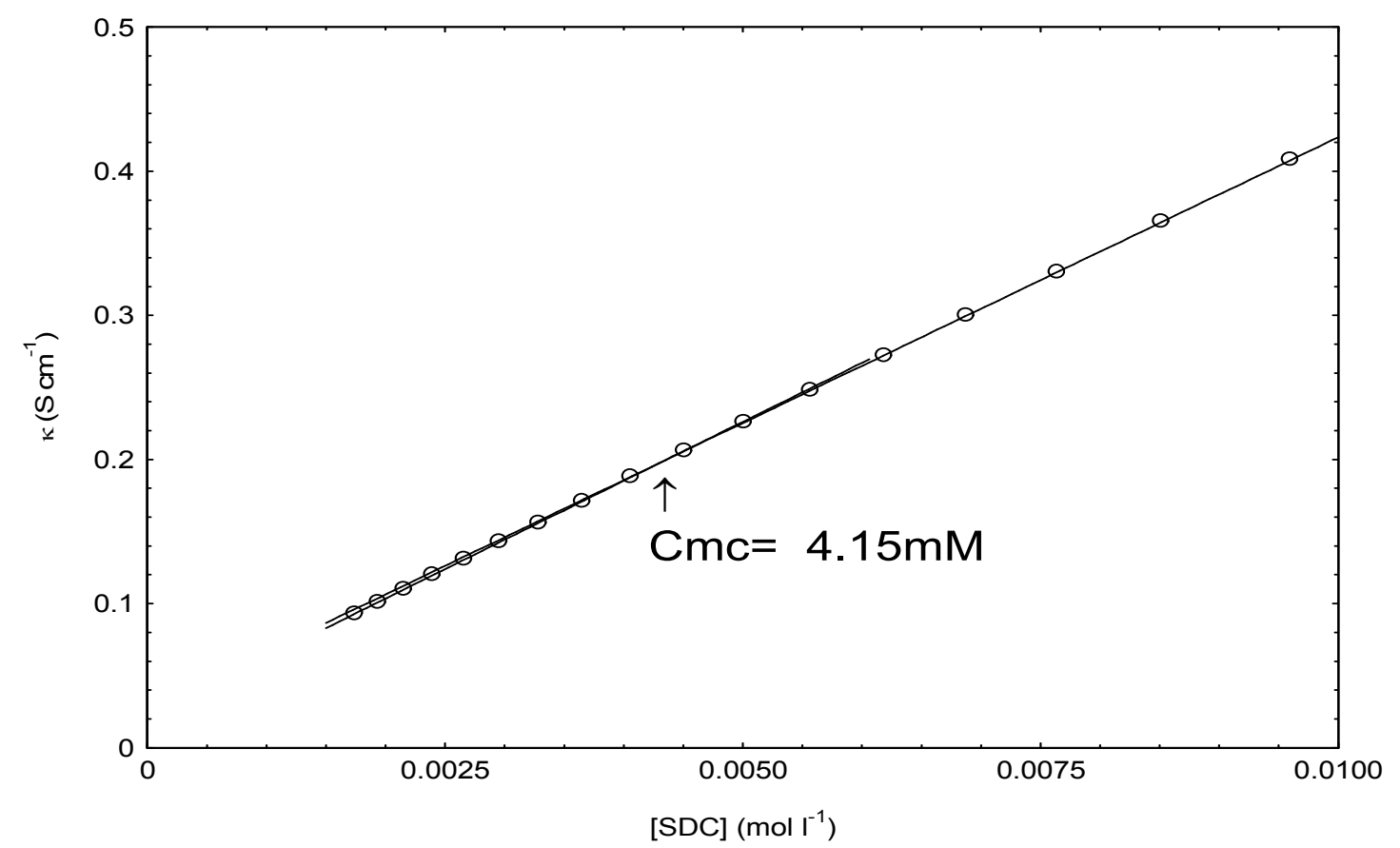

Fig. 4: Specific conductivities of SDC as a function of the surfactant concentration in $303.15 \mathrm{~K}$ : opened circles represent 0.20 volume fractions of ethanol in the solvent mixture.

\section{Conclusions}

The following conclusions have been drawn from the above results and discussion. Experimental result for the conductivity of SDC solution in pure water and ethanol- water mixed solvent media has been presented as a function of salt concentration and different percentage composition of ethanol-water mixed solvent media. The cmc of SDC are found to increase with the increase in the volume fraction of ethanol in ethanol-water mixed solvent media.

\section{Acknowledgements}

The authors are thankful to Professor Dr. Sujeet Kumar Chatterjee at the department of Chemistry, Mahendra Morang Adarsh Multiple Campus, Tribhuvan University, Biratnagar, Nepal for the valuable suggestions and discussions. The authors are also thankful to Associate Professor Ghanashyam Shrivastav, the Head of Department of Chemistry, Mahendra Morang Adarsh Multiple Campus, Tribhuvan University, Biratnagar, Nepal for providing us the research facilities to conduct this research work.

\section{References}

[1] A. D. McNaught and A. Wilkinson, Compendium of Chemical Terminology, 2nd ed. Blackwell Scientific Publications, Oxford, IUPAC (1997).

[2] A. Bhattarai, S.K. Shah and A. K. Yadav, Nepal J. Sci. and Tech.,13 (2012) 89.

[3] H. Streuli, et. al, SLMB - Schweizer Lebensmittelbuch, chapter 58, 4/3, (1992).

[4] N. A. Lange and J. A. Dean, Lange's Handbook of Chemistry, 10th ed. McGraw-Hill, New York, (1967).

[5] J .J. Lee and M. D. Lee, Korean J. Chem. Eng., 5 (1988) 5. 
A. Bhattarai et al. / BIBECHANA 9 (2013) 63-68: BMHSS, p. 68 (Online Publication: Nov., 2012)

[6] A. Bhattarai and S. K. Sah, Res. J. Chem. Sci., 1 (2011) 1.

[7] A. Bhattarai and J. Raut, J. Alpine Chemistry, 2 (2011) 44.

[8] H. S. Harned and B. B. Owen, The physical chemistry of electrolytic solutions, Reinhold, New York, (1957) 234.

[9] J. E., Jr.Lind, J. J. Zwolenik and R.M. Fuoss, J. Am. Chem. Soc., 81 (1959) 1557.

[10] B. Das and D. K. Hazra, Bull. Chem. Soc. Jpn., 65 (1992) 3470.

[11] B. Das and D. K. Hazra, J. Phys. Chem., 99 (1995) 269.

[12] S. Das, U. Thapa and K. Ismail, Bull. Chem. Soc. Jpn., 83 (2010) 1352.

[13] K. Matsuoka and Y. Moroi, Biochimica et Biophysica Acta, 1580 (2002) 189.

[14] L. R. Bogdanova $a$, O. I. Gnezdilovb, B. Z. Idiyatullin $a$, R. Kh. Kurbanova, Yu. F. Zueva, and O. G. Usyarovc, Colloid J., 74 (2012) 1.

[15] K. Gracie, D. Turner and R. Palepu, Can. J. Chem., 74 (1996) 1616.

[16] S.K.Shah, T.P. Niraula, A.Bhattarai and S.K. Chatterjee, BIBECHANA, 8 (2012) 37. 